\title{
Chondrocalcinosis: a morphofunctional study of crystal deposition in mechanically stressed shoulder soft tissues
}

\author{
Debora Burini ${ }^{1}$ \\ Sara Salucci ${ }^{1}$ \\ Francesco Fardetti $\mathrm{i}^{2,3}$ \\ Alessandro Beccarini ${ }^{2}$ \\ Vittorio Calvisi ${ }^{3}$ \\ Pietro Gobbi ${ }^{1}$ \\ Elisabetta Falcieri1 \\ Davide Curzi ${ }^{1}$ \\ 1 Department of Biomolecular Sciences, University \\ of Urbino Carlo Bo, Urbino, Italy \\ 2 Porta Sole Hospital, Perugia, Italy \\ 3 Department of Life, Health and Environmental \\ Sciences, University of L'Aquila, L'Aquila, Italy
}

\author{
Corresponding author: \\ Davide Curzi \\ Department of Biomolecular Sciences \\ University of Urbino Carlo Bo \\ Cà Le Suore St., "E. Mattei" Campus \\ 61029 Urbino, Italy \\ Tel: 0722304311 \\ E-mail: davide.curzi@uniurb.it
}

\section{Summary}

Introduction: Chondrocalcinosis is a pathological condition characterized by the presence of calcium pyrophosphate dihydrate (CPPD) crystal deposition in the soft tissues. Even if knee articular cartilage is the most involved anatomical area, different kind of tissue and joint can be affected by this disorder.

Methods: The aim of this manuscript is to analyze at histological and ultrastructural level the crystal deposition in shoulder soft tissue subjected to mechanical stress of patients affected by CPPD disease. Moreover, the cellular behavior in the same specimens has been investigated by means of transmission electron microscopy at variable distances from crystal deposits.

Results: An interesting relationship between CP. $P D$ and cellular impairment appears in humeral articular cartilage, joint capsule and long head of biceps brachii tendon sheath, where respectively chondrocytes and fibroblasts, close to crystal deposits, reveal numerous cell damages, such as chromatin condensation, dilation of organelles or cell membrane rupture.

Conclusion: Considering that cells far to the crystals are healthy, their behavior appears to be different from that of neighboring cells, then our preliminary results suggest a possible cause-effect relationship between events.

Level of evidence: basic science study.

KEY WORDS: shoulder, chondrocalcinosis, articular cartilage, long head of biceps brachii, joint capsule, calcium crystals.

\section{Introduction}

Calcium pyrophosphate dihydrate crystal deposition disease (CPPD) is an articular disorder, characterized by the presence of radiographic articular fibro- or hyaline cartilage calcifications. This condition is also called chondrocalcinosis. CPPD is the third most common form of arthritis in elderly and epidemiological studies highlight that 4 to $7 \%$ of European and USA populations are affected by chondrocalcinosis ${ }^{1,2}$. The prevalence shows no gender difference and CP. $\mathrm{PD}$ onset is strictly correlated to aging, to date identified as the most relevant risk factor together with previous joint trauma ${ }^{3}$. In fact, Neame et al. have demonstrated an increased prevalence of knee CPPD in patients aged $80-84(17.5 \%)$, respect to those aged $55-59(3.7 \%)$, without difference between man and woman ${ }^{4}$. Moreover, CPPD is frequently associated to mechanical stress induced by repetitive trauma, as confirmed by its relationship with sport practice or injuries ${ }^{5}$.

CPPD and osteoarthritis (OA) often coexist in the same joint, probably inducing a symptom worsening. The presence of calcium crystals has been observed in cartilage specimens in $25-30 \%$ of patients affected by $\mathrm{OA}$, following knee arthroplasty ${ }^{6,7}$.

The most frequent anatomical site of CPPD is knee, followed by wrist, hip, pubis symphysis and shoulder$^{8}$. From a radiological point of view, crystal deposition has been observed in the connective tissues (mainly fibrocartilage and hyaline cartilage) of different joint anatomical structures, such as menisci, articular capsule, tendon, ligament, synovial bursae, synovial fluid, synovial membrane, articular cartilage ${ }^{9}$. Meniscus fibrocartilage is the most affected tissue (from 86.3 to $95 \%$ of patients affected by CPPD in two different studies), followed by articular cartilage (from 45 to $56.8 \%$ ) and articular capsule fibrous con- 
nective tissue (about 30\%) ${ }^{4,8}$. From a histological point of view, crystal depositions in human connective tissue were found close to degenerating collagen fibers in the matrix surrounded hypertrophic chondrocytes. This kind of matrix was characterized by electron-dense amorphous material, including proteoglycans and debris of cellular components ${ }^{10}$. Moreover, crystal deposition induced fibrosis, angiogenesis and neutrophils accumulation in the ligament flavum ${ }^{11}$.

Unlike knee, epidemiological data on glenohumeral joint crystals distribution are unknown and a limited literature describes this pathological condition on shoulder tissue. Frequently it is difficult to discern CPPD and OA in this anatomical area, due to their similar radiographic features, such as subchondral bone sclerosis and osteophytes ${ }^{12}$. The presence of calcifications has been observed, by means of magnetic resonance imaging and $\mathrm{X}$-rays technique, in humeral articular cartilage, tendons, capsule, synovial fluid, synovial membrane and bursae ${ }^{13,14}$.

Although the mechanisms of connective tissue calcification are not completely understood even for the most common and studied crystal deposition diseases, an impaired chondrocyte behavior seems to be involved in articular cartilage calcification of patients affected by $O A^{15}$. In particular, similarly to other kinds of crystals, calcium pyrophosphate dihydrate precipitates may induce cell death, involving the necroptosis pathway ${ }^{16}$.

The aim of this work was to investigate at microscopic and histological level the crystal deposition on shoulder soft tissues of patients affected by CPPD. Electron microscopy analysis has been performed in the same specimens to investigate the cellular behavior close to crystal and far from them. This manuscript allows correlate the crystal microscopic location and cell behavior, suggesting an interesting relationship.

\section{Materials and methods}

\section{Patients and specimen handling}

The study meets the ethical standards of the journal. In particular, all experimental procedures were carried out according to the journal guidelines ${ }^{17}$.

Specimens from humeral articular cartilage, joint capsule, long head of biceps brachii and loose bodies were withdrawn from 6 patients with CPPD, aged $67 \pm 5$, during shoulder arthroplasty. For each patient, pre-surgery X-ray images have been performed to confirm the typical degenerative features of CPPD. In particular, shoulders revealed a diffuse calcium crystal deposit in the soft tissue (Figs. 1A, B). Fragments
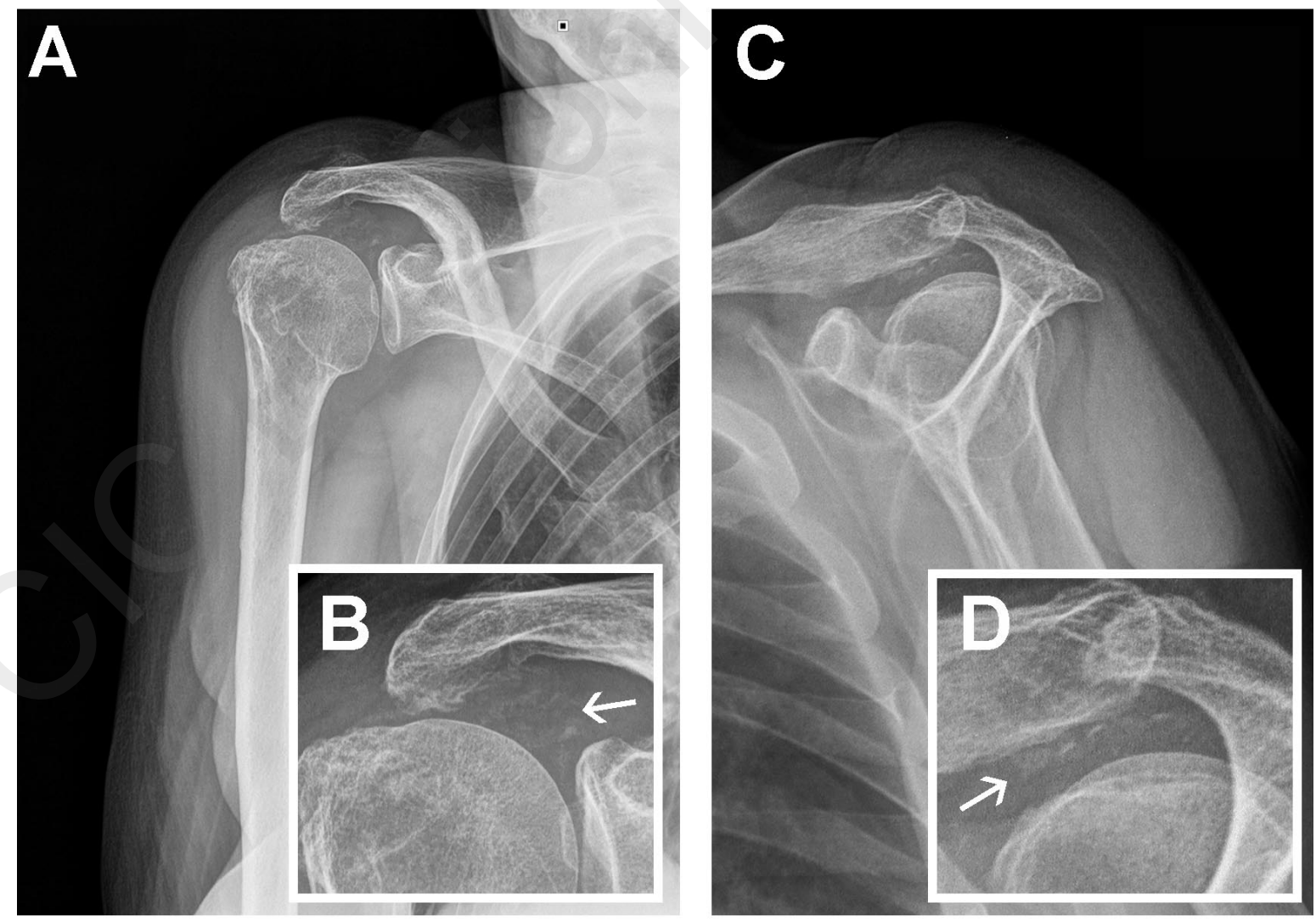

Figure 1. Radiographs of right and left shoulders of patients affected by CPPD: anteroposterior (A) and outlet-view (C). At high magnification $(B, D)$, the presence of widespread calcium crystal accumulations in soft tissues is observable (arrow). 
from each tissue were fixed with $2.5 \%$ glutaraldehyde solution in a $0.1 \mathrm{M}$ phosphate buffer for $3 \mathrm{~h}$ and then minced into smaller specimens $\left(3 \mathrm{~mm}^{3}\right)$, which were fixed in the same solution for a supplementary hour. After washing, samples were post-fixed with $1 \%$ osmium tetroxide solution in the same buffer for $1 \mathrm{~h}$, rinsed and dehydrated in a graded series of ethanol ${ }^{18}$.

\section{Environmental scanning electron microscopy (ESEM) and microanalysis}

Fixed samples from each tissue were dried and mounted on conventional SEM stubs, and then observed with a FEI QUANTA 200 environmental scanning electron microscope (mode: low vacuum; detector: secondary electrons; high voltage: $30 \mathrm{kV}$ ). To confirm the diagnosis of chondrocalcinosis, an energy dispersive X-ray spectroscopic microanalysis (EDS) has been performed in the same conditions of observation ${ }^{19}$

\section{Histological analysis}

Other fixed fragments were embedded in araldite and sectioned by an LKB 2088 ultramicrotome. Semithin sections were stained with $1 \%$ toluidine blue in distilled water for 2 minutes. After washing, they were stained with $2 \%$ alizarin red in distilled water for 2 minutes. To better fix alizarin red staining, specimen sections were immersed for 20 seconds in acetone, acetone-xylene (1:1) solution and xylene, respectively. Then, samples were washed and observed by means of Olympus optical microscope at 10x, 20x, 40x or $100 x($ oil) 20 .

\section{Transmission electron microscopy (TEM)}

The stained semithin sections were used to identify areas where to perform TEM analysis. Thin sections were then obtained from these chosen regions, stained with uranyl acetate and lead citrate solutions and then observed with Philips CM10 transmission electron microscope (voltage: $80 \mathrm{kV})^{21}$.

\section{Results}

\section{Humeral articular cartilage}

Chondrocalcinotic humeral articular cartilage displayed a general degradation. In transverse sections (Fig. 2A), numerous clefts occurred from the articular surface to the deeper tissue layers, and some areas of cartilage surface layer appeared completely destroyed. Calcium crystal depositions (red stained) were visible on the superficial zones, as well as in the middle layers of tissue (Fig. 2B). These crystals appear to be generally collected in deposits and rarely can be found alone. When this happens, it affects the deeper levels of the tissue and not the superficial ones.

The cellular distribution was deeply modified, as confirmed by the almost complete absence of cells on the superficial layers. In the middle zone, TEM im- ages revealed the presence of rare healthy chondrocytes, which showed a regular organization of nucleus, nuclear pores and chromatin, as well a diffuse endoplasmic reticulum and numerous glycogen granules (Fig. 2C). However, several chondrocytes displayed necrotic features, such as vacuolized cytoplasm, degraded organelles, plasma and nuclear membrane discontinuities, with lack of chromatin condensation (Fig. 2D).

\section{Long head of biceps brachii tendon sheath}

Optical images of the long head of biceps brachii tendon revealed a high tissue mineralization. In particular, the outer fibrous sheath, that, together with the inner synovial ones, forms the tendon sheath, appeared characterized by crystal accumulations, as well as numerous single crystals scattered in the tissue. Analyzing at optical microscopy the tissue areas close to these sediments, fibroblasts showed an evident vacuolization, suggesting a compromised cell vitality (Fig. 3A). TEM micrographs confirmed the impaired cell morphology. Fibroblasts showed an evident cell shrinkage with visible cytoplasmic alterations characterized by numerous vacuoles, many of which containing cellular material inside. In addition, chromatin condensation appeared (Fig. 3C). On the other hand, tissue areas far from crystal deposit showed preserved healthy cells (Fig. 3B). In most of these cells, the cytoplasmic vacuoles were completely absent and, when present, they had a clear reduced dimension if compared to those close to crystal sediments.

\section{Joint capsule}

Optical transverse sections of articular capsule displayed wide crystal accumulations on the synovial membrane, and the presence of single crystals scattered within the tissue (Fig. 4A, B). The capsule integrity appeared compromised by tissue clefts, extending from the joint cavity to the tissue deeper layers. From optical images, a relationship between single crystal and clefts locations was observable. In particular, the sediments in the depth layers of tissue appeared neighboring to the cleft wall.

At ultrastructural level, the fibroblast-like synoviocyte, if compared to few preserved cells (Fig. 4C), revealed the presence of autophagic vacuoles and swollen organelles (Fig. 4D).

\section{Loose body}

Optical microscope images revealed wide calcified tissue area in the intra-articular loose body. Given the nature of this anatomical structure, which is usually composed by cartilage or cartilage and bone, the calcification presence is apparently not noteworthy ${ }^{22}$. However, given the pathology of the patient, understanding the nature of these crystals was necessary and the crystal analysis confirmed results comparable to bone tissue (data not shown). Morphological observations were not able to identify single or accumulated calcium pyrophosphate dihydrate crystals in this 

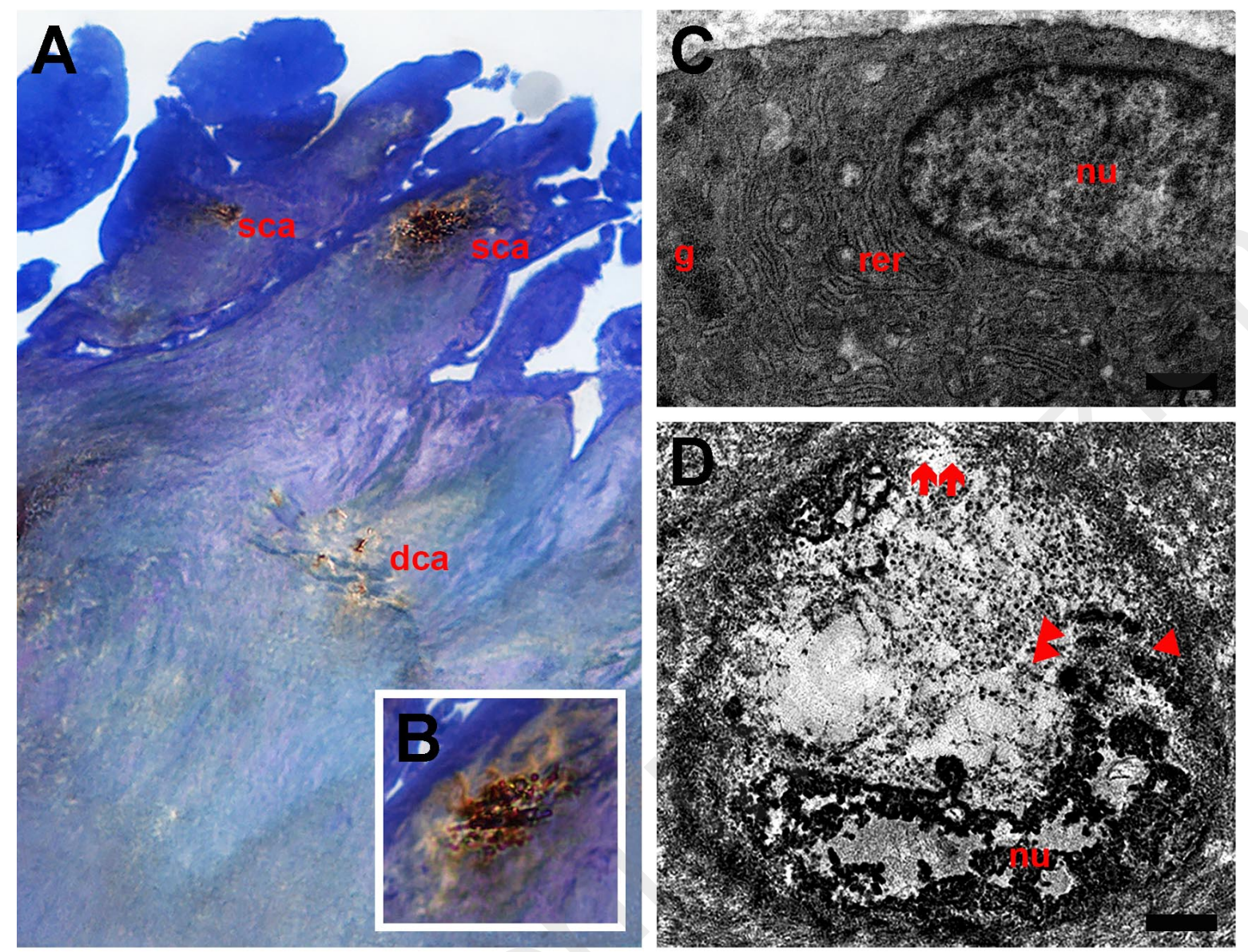

Figure 2. Light microscopy (A, B) and TEM (C, D) images of chondrocalcinotic cartilage cross-sections. In $A$ and $B$ the accumulations of CPPD crystals appear red stained. In A, red stained superficial (sca) and deep crystal accumulations (dca) are observable. TEM micrographs of cartilage middle layers reveal preserved chondrocytes far to crystal deposits, which show an undamaged nucleus (nu), an abundant rough endoplasmic reticulum (rer) and several glycogen granules (g). Close to crystal deposits, necrotic chondrocytes with discontinuous nuclear (arrow heads) and plasma membrane (arrows), appear. Magnification: A: 20x; B: 100x. Bars: C, D: $1 \mu \mathrm{m}$.

tissue (Fig. 5A), however in the high calcified zones the identification of specific kinds of crystals appeared really hard. Even though the loose body is a free fragment in the articular space, its cells showed a high vitality if compared to those of other tissues. TEM micrographs confirmed the chondrocyte morphofunctional integrity. In particular, cells showed a regular arrangement of nuclear and cytoplasmic components (Fig. 5B).

\section{Calcium pyrophosphate dihydrate crystal deposition}

Single crystal ultrastructure and accumulation morphology were respectively evaluated by ESEM and TEM in each tissue (Fig. 6A, B). The crystal chemical nature was investigated by EDS spectrum (Fig. 6C), showing the relationship between calcium and phosphorous peaks, in line with the stoichiometric ratio of CPPD crystals.

\section{Discussion}

Although the diagnoses of CPPD of shoulder soft tissues are increasing, many questions regarding this pathological condition, such as its origin or its development, still have no answers. Numerous scientific works have analyzed this condition at macroscopic level through radiological investigation, but, to the best of our knowledge, this is the first study which examined, by means of various microscopy techniques (previous described), CPPD crystal deposition in different human shoulder anatomical structures, evaluating a possible relationship between cell behavior and crystal deposits location.

If cell death is the origin or the consequence of crystal deposition is still under discussion. The molecular mechanisms of CPPD crystal formation have not yet been fully understood, but the most quoted hypothesis in human cartilage argues that chondrocyte dys- 

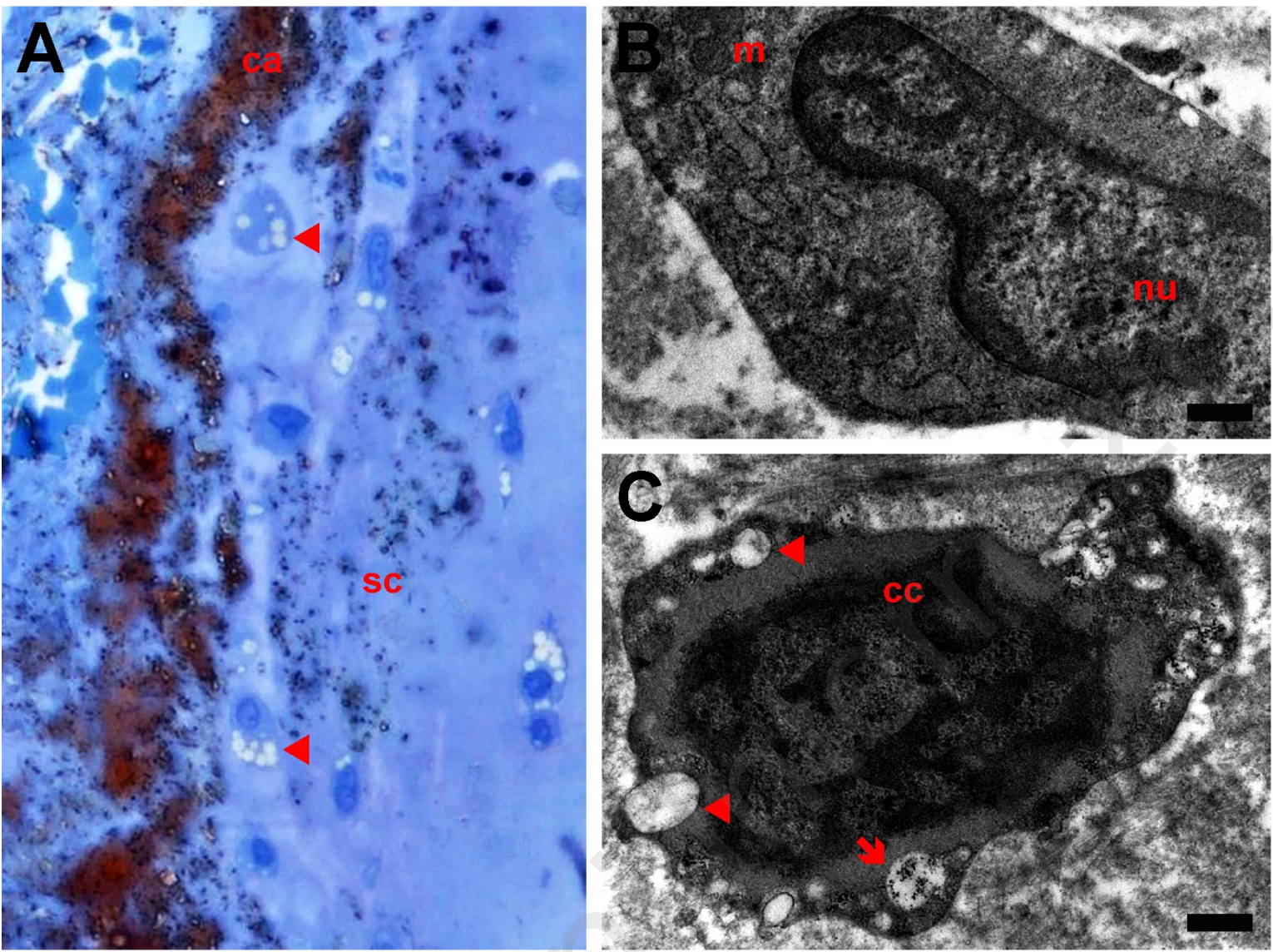

Figure 3. Light microscopy (A) and TEM (B, C) images of the outer fibrous layer of long head of biceps brachii tendon sheath. In A, red stained crystal accumulations (ca) and single crystal (sc) are observable near to high vacuolated cells (arrow head). At high magnification, fibroblasts far to crystal deposits display a healthy morphology (B), characterized by a regular disposition of chromatin in the nucleus ( $\mathrm{nu}$ ) and undamaged mitochondria $(\mathrm{m})$. On the other hand, cells close to sediments show a compromised ultrastructure $(\mathrm{C})$, with a starting chromatin condensation and numerous vacuoles in cytoplasm (arrow head), some of which seems containing cellular material inside (arrow). Magnification: A: 40x. Bars: B, C: 0,5 $\mu \mathrm{m}$.

function in maintaining extracellular matrix turnover would be the starting point. In particular, these cells seem to produce excessive extracellular inorganic pyrophosphate in the tissue. These molecules are stored in synovial fluid through the direct contact between tissues and the weeping lubrication mechanism of cartilage. In the synovia, a significant increase of inorganic pyrophosphate induces CPPD crystal deposits $2,3,22$, which in turn are allocated, as shown by our results, in the different soft tissues in direct or indirect contact with the synovial fluid, such as humeral articular cartilage, inner synovial membrane of joint capsule and long head of biceps brachii tendon sheath. Our preliminary results suggest a key role of tissue wear, related to mechanical stress and to crystal deposits themselves. In fact, even if the calcification process may develop within the same tissue, as well as for other pathological conditions, these results reveal a clear relationship between crystal location and tissue clefts, suggesting the ability of these crystals to insert themselves into the clefts through the synovial fluid, and then their capacity to pass from that space to the close tissue areas. Compared to other shoulder soft tissue, morphological and chemical techniques were not able to reveal CP. PD crystals in the loose body. Nevertheless, the osteochondral nature of this structure could have affected our analysis. In particular, the identification of CPPD crystals in the large calcified area appeared particularly complex. According to the literature ${ }^{23}$, despite the loose body is a free fragment in the joint cavity, its cartilaginous area was characterized by numerous healthy chondrocytes, confirming the synovial fluid ability to regularly make contact with it. Although our observations have not confirmed it, being the synovial fluid the carrier of crystals, this contact suggests a probable crystals deposit in this tissue.

The crystal deposition effects on each tissue are not yet clear. Some studies suggest a relationship between them and acute inflammation, which probably depends on the crystals interaction with neutrophils in the synovial fluid24,25. CPPD crystal accumulation in articular cartilage could also alter its mechanical properties, contributing to joint damage ${ }^{2}$. There are 

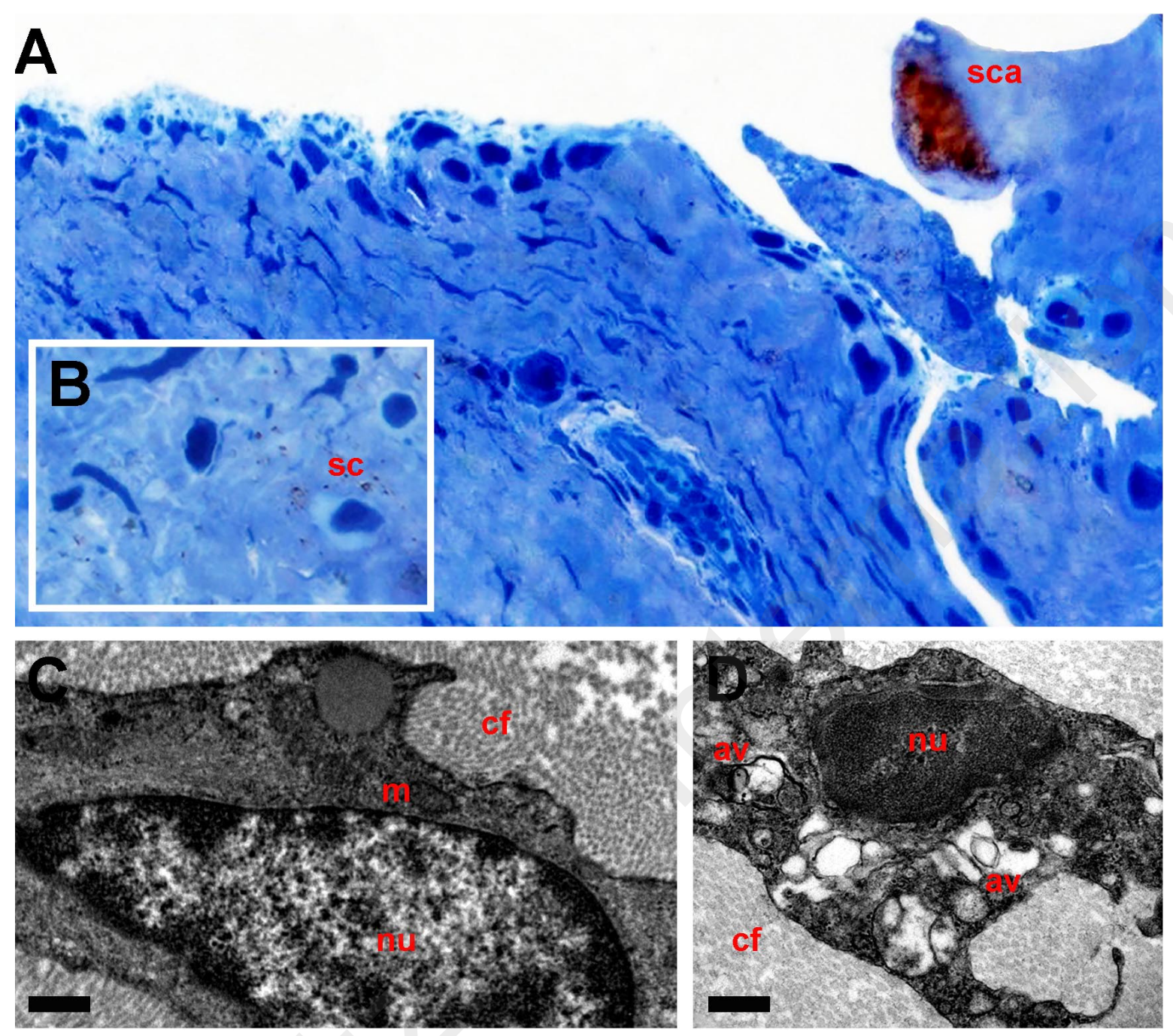

Figure 4. Light microscopy (A, B) and TEM (C, D) images of synovial membrane and joint capsule. In A, red stained superficial crystal accumulation (sca) appears at the synovial membrane, while in B, the presence of single crystals (sc) in the deeper layers of joint capsule is observable. Synoviocytes, supported by collagen fibers (cf), show a regular chromatin disposition in the nucleus (nu), far from sediments, as well as undamaged mitochondria in the cytoplasm. Contrary, synoviocytes close to crystal deposits display an electron dense nucleus and numerous autophagic vacuoles (av) in the cytoplasm, suggesting a compromised cell vitality. Magnification: A: 20x; B: 40x. Bars: C, D: 0,5 $\mu \mathrm{m}$.

no evidences, instead, about a possible cell death associated to CPPD. There are only rare studies referred to other pathologies, for instance calcium oxalate stones seem to induce death in renal epithelial cells, but the mechanism is unclear ${ }^{25}$.

In this study, a relationship between crystal sediments location and cell death has been observed. In humeral cartilage superficial zone, where calcium deposits are more significant, an almost total absence of chondrocyte is observable. Even if their death can be easy related to tissue wear, near single CPPD crystals, in cartilage middle layer numerous necrotic cells have been revealed. Similarly, in the long head of biceps brachii tendon sheath, impaired and healthy fibroblasts are major concentrated, respectively, close and far away from the calcium crystals. The synovial membrane of joint capsule displayed a comparable condition, where several impaired cells are specifically located close to CPPD crystal accumulations. Taken together, these preliminary findings suggest a connection between cell death and CPPD crystals. However, in order to understand their cause and effect relationship, future studies will be necessary and the possible existence of a positive feedback system between the two events should be considered as a hypothesis worth examining.

\section{Compliance with ethical standards}

\section{Funding}

The Authors received no specific funding for this 

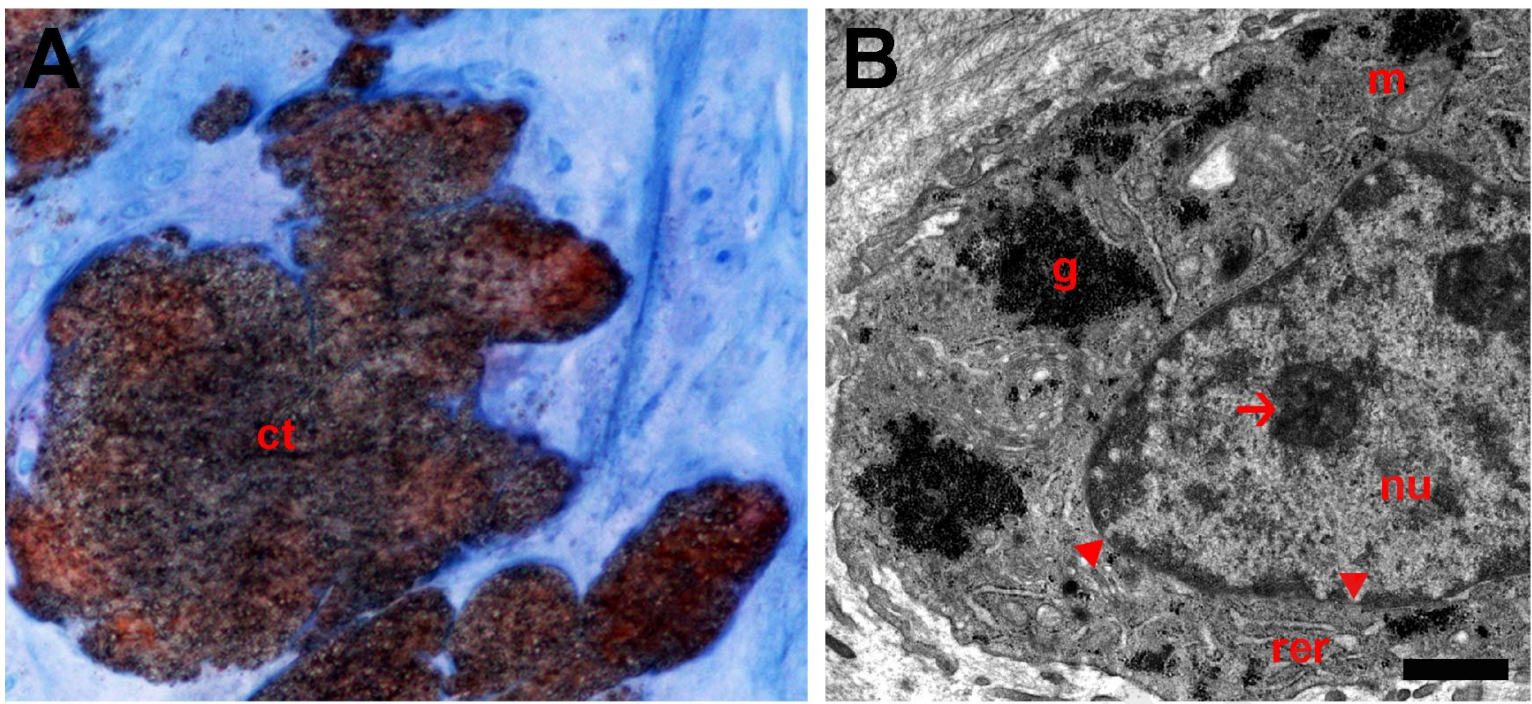

Figure 5. Light microscopy (A) and TEM (B) images of loose body. Numerous and wide calcified area (ca) from bone origin (red stained) surrounded from cartilaginous tissue are observable (A). A preserved chondrocyte appears, displaying an undamaged nucleus ( $\mathrm{nu}$ ) and nucleolus ( $\mathrm{n}$ ) and, in the cytoplasm, a high concentration of glycogen granules (g) and rough endoplasmic reticulum (rer) is observable. Magnification: A: 20x. Bars: B: $1 \mu \mathrm{m}$.
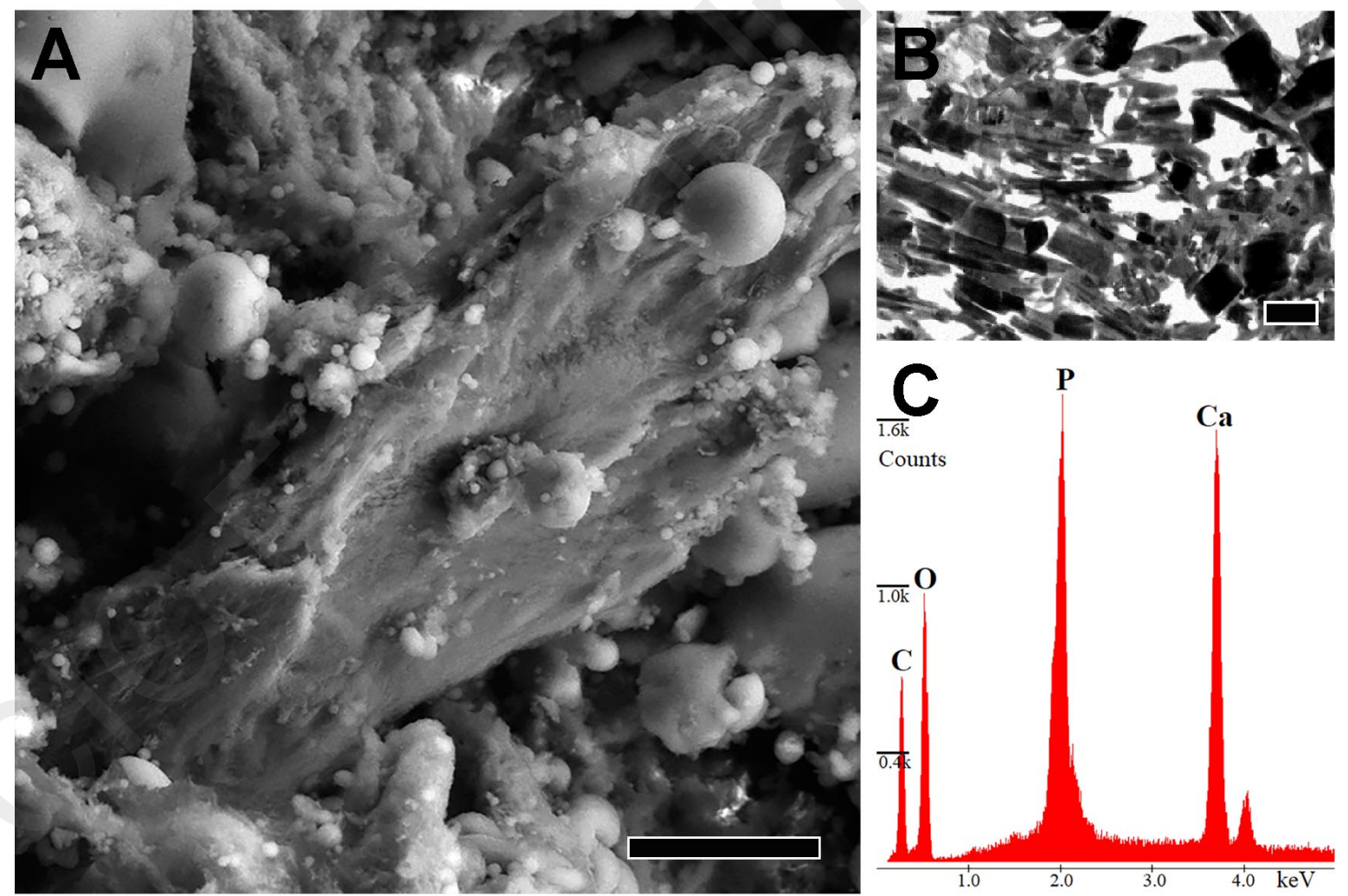

Figure 6. ESEM (A) and TEM (B) images of a single calcium pyrophosphate dihydrate crystal and an accumulation of numerous crystals in cartilaginous tissue respectively. In C, EDS spectrum confirms the nature of crystals. Bars: A: $2 \mu \mathrm{m}$; B: 5 $\mu \mathrm{m}$.

work, but the work was only supported by "Fondi Valorizzazione" and "Fondi Ricerca" DiSB, Carlo Bo
Urbino University, which are a form of personal research funds. 


\section{Conflict of interest}

The Authors declare that they have no conflict of interest.

\section{Acknowledgements}

We wish to thank Dr. Rita De Matteis and Dr. Sabrina Burattini from Department of Biomolecular Sciences of Urbino University, for their advice in histological analysis and technical help respectively. Also thank to Dr. Patrizia Ammazzalorso, head of Pesaro ARPAM department, for ESEM analysis assistance. This work has been possible thanks to the DISB 2017 Enhancement Project of Urbino University.

\section{References}

1. Ferrone C, Andracco R, Cimmino MA. Calcium pyrophosphate deposition disease: clinical manifestations. Reumatismo. 2011;63:246-252.

2. Rosenthal AK, Ryan LM. Calcium Pyrophosphate Deposition Disease. N Engl J Med. 2016;374:2575-2584.

3. Sivera F, Andres M, Pascual E. Calcium pyrophosphate crystal deposition. Int J Clin Rheumatol. 2011;6:677-688.

4. Neame RL, Carr AJ, Muir K, Doherty M. UK community prevalence of knee chondrocalcinosis: evidence that correlation with osteoarthritis is through a shared association with osteophyte. Ann Rheum Dis. 2003;62:513-518.

5. Jennings F, Lambert E, Fredericson M. Rheumatic diseases presenting as sports-related injuries. Sports Med. 2008;38 917-930.

6. Derfus BA, Kurian JB, Butler JJ, et al. The high prevalence of pathologic calcium crystals in preoperative knees. J Rheumatol. 2002;29:570-574.

7. Viriyavejkul P, Wilairatana V, Tanavalee A, Jaovisidha K. Comparison of characteristics of patients with and without calcium pyrophosphate dihydrate crystal deposition disease who underwent total knee replacement surgery for osteoarthritis. Osteoarthritis Cartilage. 2007;15:232-235.

8. Abhishek A, Doherty S, Maciewicz R, Muir K, Zhang W, Doherty M. Chondrocalcinosis is common in the absence of knee involvement. Arthritis Res Ther. 2012;14:R205.

9. Sussmann AR, Cohen J, Nomikos GC, Schweitzer ME. Magnetic resonance imaging of shoulder arthropathies. Magn Reson Imaging Clin N Am. 2012;20:349-371.

10. Ishikawa K, Masuda I, Ohira T, Yokoyama M. A histological study of calcium pyrophosphate dihydrate crystal-deposition disease. J Bone Joint Surg Am. 1989;71:875-886.
11. Yayama T, Baba H, Furusawa N, et al. Pathogenesis of calcium crystal deposition in the ligamentum flavum correlates with lumbar spinal canal stenosis. Clin Exp Rheumatol. 2005;23:637-643.

12. Magarelli N, Amelia R, Melillo N, Nasuto M, Cantatore F, Guglielmi G. Imaging of chondrocalcinosis: calcium pyrophosphate dihydrate (CPPD) crystal deposition disease - imaging of common sites of involvement. Clin Exp Rheumatol. 2012; 30:118-125.

13. Huang GS, Bachmann D, Taylor JA, Marcelis $S$, Haghighi $P$, Resnick D. Calcium pyrophosphate dihydrate crystal deposition disease and pseudogout of the acromioclavicular joint: radiographic and pathologic features. J Rheumatol. 1993;20: 2077-2082

14. Bencardino JT, Hassankhani A. Calcium pyrophosphate dihydrate crystal deposition disease. Semin Musculoskelet Radiol. 2003;7:175-185.

15. Ea HK, Lioté F. Advances in understanding calcium-containing crystal disease. Curr Opin Rheumatol. 2009;21:150-157.

16. Mulay SR, Desai J, Kumar SV, et al. Cytotoxicity of crystals involves RIPK3-MLKL-mediated necroptosis. Nat Commun. 2016;7:10274.

17. Padulo J, Oliva F, Frizziero A, Maffulli N. Muscles, Ligaments and Tendons Journal - Basic principles and recommendations in clinical and field science research: 2016 update. MLTJ. 2016;6(1):1-5.

18. Curzi $D$, Ambrogini $P$, Falcieri E, Burattini S. Morphogenesis of rat myotendinous junction. MLTJ. 2014;3:275-280.

19. Curzi D, Fardetti F, Beccarini A, et al. Chondroptotic chondrocytes in the loaded area of chondrocalcinotic cartilage: A clinical proposal? Clin Anat. 2017. [Epub ahead of print]

20. Yamakawa K, Iwasaki $\mathrm{H}$, Masuda I, et al. The utility of alizarin red s staining in calcium pyrophosphate dihydrate crystal deposition disease. J Rheumatol. 2003;30:1032-1035.

21. Curzi D, Baldassarri V, De Matteis R, et al. Morphological adaptation and protein modulation of myotendinous junction following moderate aerobic training. Histol Histopathol. 2015;30:465-472.

22. Melrose J. The knee joint loose body as a source of viable autologous human chondrocytes. Eur J Histochem. 2016;60 :2645.

23. Abhishek A, Doherty M. Pathophysiology of articular chondrocalcinosis - role of ANKH. Nat Rev Rheumatol. 2011;7: 96-104.

24. Tudan C, Jackson JK, Higo TT, Hampong M, Pelech SL, Burt HM. Calcium pyrophosphate dihydrate crystal associated induction of neutrophil activation and repression of TNF-alphainduced apoptosis is mediated by the p38 MAP kinase. Cell Signal. 2004;16:211-221.

25. Sun $X Y$, Gan QZ, Ouyang JM. Calcium oxalate toxicity in renal epithelial cells: the mediation of crystal size on cell death mode. Cell Death Discov. 2015;1:15055. 